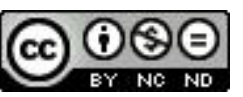

\title{
Precisión de los Estudiantes de Psicología en la Estimación de la Asociación*
}

\section{Psychology Student's Estimation of Association}

\author{
Gustavo R. Cañadas** \\ Carmen Díaz*** \\ Carmen Batanero**** \\ Antonio Estepa ${ }^{* * * * *}$
}

\begin{abstract}
Resumen
Las tablas de contingencia son un recurso frecuente para presentar información estadística en prensa e Internet, así como en documentos técnicos usados en el trabajo profesional, aunque la enseñanza actual no presta mucha importancia a este tema, suponiendo que su interpretación es sencilla. En este trabajo analizamos la percepción de la asociación y estimación de su intensidad en tablas de contingencia en una muestra de 414 estudiantes de psicología de tres universidades españolas. Se observan respuesta similares en las tres universidades, alta consistencia entre percepción subjetiva de la asociación y precisión de la estimación y mejores resultados que los obtenidos en un estudio previo de Estepa (1994) con estudiantes de Bachillerato. Sin embargo, las estrategias al realizar el juicio de asociación son mayoritariamente incorrectas y sólo

\footnotetext{
*Agradecimientos: este trabajo forma parte de la beca FPU AP2009-2807 (MEC-FEDER), proyecto EDU2010-14947 (MICIN) y Grupo PAI FQM126. Junta de Andalucía.

** Maestría en Estadística/Universidad de Granada, España. Profesor Sustituto Interino del Departamento de Didáctica de la Matemática. Facultad de Educación, Universidad de Granada. Dirección de envio: Campus de Cartuja, 18071, Granada, España. E-mail: grcanadas@ugr.es.

*** Doctora en Metodología de las Ciencias del Comportamiento, Universidad de Huelva. Profesor Contratado Doctor, Departamento de Psicología, Facultad de Educación y Psicología, Universidad de Huelva. Dirección de envio: Campus El Carmen, Avda. Fuerzas Armadas, 21071 H21071 Huelva, España.E-mail: carmen.diaz@dpsi.uhu.es.

${ }^{* * * * *}$ Doctorado en Matemáticas/Universidad de Granada, España. Catedrática de Universidad del Departamento de Didáctica de la Matemática. Facultad de Educación, Universidad de Granada. Dirección de envio: Campus de Cartuja. 18071, Granada, España, E-mail: batanero@ugr.es.

****** Doctorado en Didáctica/Universidad de Granada, España. Catedrático de Universidad del Departamento de Didáctica de las Ciencias. Facultad de H. y Ciencias de la Educación, Universidad de Jaén. Dirección de envio: Campus las Lagunillas, 23071, Jaén, España, E-mail: aestepa@ujaen.es.
} 
influyen en la precisión del juicio en el caso de independencia en los datos. Estos problemas podrían presentarse en otros estudiantes, por lo que los profesores debieran conocerlos para tenerlos en cuenta en la enseñanza.

Palabras-clave: Tablas de Contingencia. Asociación. Estimación de la Asociación. Teorías Previas.Enseñanza Universitaria.

\begin{abstract}
Contingency tables are frequently used to present statistical information in the media and the Internet, as well as in technical documents used for professional work. However, they are rarely taken into account in the teaching of statistics, since their interpretation is considered easy. In this paper, we analyse the perception of association and the estimation of its intensity in contingency tables in a sample of psychology students. We observe similar responses in the three universities in the sample, and high consistency between the subjective perception of association and the accuracy of estimates, as well as better results than those in a previous study of Estepa (1994) carried out with preuniversity students. However, the strategies used to judge the association are mostly incorrect and only influence the accuracy of the estimate in case of independence of the data. These problems may also be common in other students; consequently teachers should know these results to take them into account in their teaching.
\end{abstract}

Keywords: Contingency Tables. Association. Estimating Association. Previous Theories. University Teaching.

\title{
1 Introducción
}

Un modo común de presentar la información estadística es en forma de tabla de doble entrada o tabla de contingencia, a la que se presta poca atención en la enseñanza universitaria, suponiendo que su lectura e interpretación son sencillas. Este tipo de tablas puede encontrarse en muchos contextos, como en la prensa o Internet, y pueden utilizarse como instrumento de análisis de datos, así como para retener en la memoria una gran cantidad de información en forma eficiente (CAZORLA, 2002). Tienen un papel esencial en la organización, descripción y análisis de datos, al ser un instrumento de transnumeración, forma básica de razonamiento estadístico que proporciona nueva información, al cambiar de un sistema de representación a otro (WILD; PFANNKUCH, 1999).

Dichas tablas se presentan, además, con frecuencia, en actividades profesionales; por ejemplo, en el diagnóstico médico o psicológico, donde el especialista se enfrenta a diferentes síntomas que pueden estar asociados o no 
con una patología (DÍAZ; GALLEGO, 2006). Por otro lado, el razonamiento sobre datos bivariados es uno de los temas de aprendizaje prioritarios en los cursos de estadística en la universidad y los últimos años de educación secundaria (ZIEFFLER, 2006). Por otro lado, la asociación estadística extiende, además, la idea de dependencia funcional, y se utiliza en muchos métodos de gran variedad de ciencias, por su relación con la toma de decisiones en ambiente de incertidumbre. Es por ello difícil imaginar una actividad cognitiva más importante que el razonamiento sobre datos bivariados (MCKENZIE; MIKKELSEN, 2007), ya que la habilidad para evaluar adecuadamente la asociación permite a las personas explicar el pasado, controlar el presente y predecir el futuro (CROCKER, 1981).

En consecuencia, el estudio de los sesgos de razonamiento de los estudiantes al interpretar las tablas de contingencia es un tema de investigación de interés para la educación matemática, pues los profesores que se enfrentan a la enseñanza del tema necesitan esta información, para ayudar a sus estudiantes a superar tales problemas. Para contribuir a esta problemática, el objetivo de este trabajo es evaluar la comprensión intuitiva de los estudiantes, que ingresan en la Licenciatura de Psicología, sobre la asociación en tablas de contingencia. Más concretamente, pretendemos estudiar la precisión con que se estiman los coeficientes de asociación en función de diversas variables.

\section{Investigaciones previas}

\subsection{Desarrollo del concepto de asociación}

Las investigaciones iniciales sobre este tema las inician Inhelder y Piaget (1955), quienes conciben la asociación como último paso en el desarrollo del razonamiento probabilístico y describen las estrategias usadas en los juicios de asociación en tablas 2x2 similares a la Tabla 1 a diferentes edades. Cuando se pide estudiar la posible asociación entre las variables A y B, a partir de los datos de la tabla, los sujetos al alcanzar la adolescencia comienzan usando sólo la celda $a$ para juzgar la asociación; es decir, consideran que hay asociación sólo si el número de casos en que se presentan a la vez A y B (celda $a$ en la tabla) es suficientemente elevado.

Entre los 12-15 años, los alumnos solamente comparan celdas dos a dos (por ejemplo comparan la celda $a$ con la celda $b$ ), sin entender que las celdas $a$ y $d$ tienen el mismo peso en relación a la asociación. Otro nivel posterior sería 
comprender cuales son los casos favorables $(a$ y $d)$ y desfavorables $(b$ y $c)$ a la asociación, sin compararlos. Finalmente, se establecen las relaciones diagonales ( $a$ y $d$ se perciben como favorables a la asociación, mientras que $b$ y $c$ serían contrarias), comparándolas entre sí o con el total $(a+b+c+d)$. Jenkins y Ward (1965) indican que la estrategia de comparar las diagonales sólo se puede usar con frecuencias marginales iguales para la variable independiente, y proponen, como estrategia correcta para casos generales, comparar la diferencia entre las probabilidades $P(B \mid A)$ y $\mathrm{P}(B \mid n o A)$.

Tabla 1- Esquema de una tabla de contingencia $2 \times 2$

\begin{tabular}{cccc}
\hline & $B$ & No $B$ & Total \\
\hline$A$ & $\mathrm{a}$ & $\mathrm{b}$ & $\mathrm{a}+\mathrm{b}$ \\
No $A$ & $\mathrm{c}$ & $\mathrm{d}$ & $\mathrm{c}+\mathrm{d}$ \\
Total & $\mathrm{a}+\mathrm{c}$ & $\mathrm{b}+\mathrm{d}$ & \\
\hline
\end{tabular}

\subsection{Precisión en la estimación del coeficiente de asociación}

Otros investigadores estudian la precisión en la estimación del coeficiente de asociación y las variables que lo afectan. Crocker (1981) indica que los sujetos incrementan su exactitud cuando los datos se presentan en forma de tablas; los sucesos que covarían lo hacen juntos en el tiempo; los datos no son ambiguos y se elimina la necesidad de recordar casos o estimar frecuencias.

Allan y Jenkins (1983) se centran en dos principios, en los que se basan los patrones de los juicios de asociación: (1) se tiende a basar los juicios en la diferencia entre casos en que se dan simultáneamente los dos caracteres (celda $a$ en la Tabla 1) o ninguno (celda $d$ ), y (2) la compatibilidad causal entre las variables independiente y dependiente juega un papel crítico. En el trabajo de Erlick y Mills (1967) se expone que la asociación negativa se estima como muy próxima a cero. Además, aparecen tres factores que influyen en los juicios de asociación en el trabajo de Arkes y Harkness (1983), que son: (1) el valor de la frecuencia en la casilla $a$ (Tabla 1) parece tener mayor impacto en las contingencias estimadas; (2) la etiquetación de las filas y columnas puede drásticamente influenciar las contingencias estimadas, y (3) la presencia de números pequeños en las casillas puede influir en una sobrestimación.

Chapman (1967, p.151) describe un razonamiento común, que denomina correlación ilusoria y que fue propuesto para "designar la correlación que 
perciben los observadores entre dos clases de eventos que, en realidad, (a) no están correlacionados, o (b) se correlacionan con un menor grado del que se declara, o (c) se correlacionan en la dirección opuesta de la que se declara".

Otros autores han estudiado la influencia de las teorías previas sobre el contexto del problema en la exactitud de la estimación de la asociación (JENNINGS; AMABILE; ROSS, 1982; ALLOY; TABACHNICK, 1984; WRIGHT; MURPHY, 1984; MEISER; HEWSTONE, 2006). La estimación de la asociación es más precisa si las personas no tienen teorías respecto al tipo de asociación sobre los datos. Si las teorías previas del sujeto indican el mismo tipo de asociación que reflejan los datos empíricos, los sujetos tienden a sobrestimar el valor del coeficiente de asociación. Pero, cuando los datos no reflejan los resultados esperados por estas teorías, aparece en los sujetos un conflicto cognitivo y se suelen guiar por sus teorías, más que por los datos. Así, puede resultar una estimación menor o mayor del coeficiente de asociación, dependiendo de los conocimientos previos sobre la situación que tengan los sujetos y de los datos proporcionados.

La asociación entre variables puede ser debida a la existencia de una relación causa-efecto unilateral (una variable es causa de la otra), pero, también, según Barbancho (1973), a la interdependencia (cada variable afecta a la otra), dependencia indirecta (una tercera variable afecta a otras dos), concordancia (coincidencia en preferencia u ordenación de la misma serie de datos por dos jueces) y covariación espúrea o casual. La comprensión de la asociación implicaría, además de la exactitud en el juicio, comprender estos tipos de relaciones entre las variables. Sin embargo, Estepa (1994), quien estudia las concepciones que muestran los sujetos respecto a la asociación, describe la concepción causal, según la cual el sujeto sólo considera la asociación entre variables si puede atribuirse a la presencia de una relación de causa - efecto entre las mismas. También define la concepción unidireccional como el caso en que el estudiante no admite la asociación inversa, considerándose la intensidad de la asociación, pero no su signo, y considera la asociación inversa como independencia. En un estudio posterior (BATANERO; ESTEPA; GODINO, 1997) muestran que la concepción causal no mejora con la enseñanza. Nuestro trabajo continúa el de estos últimos autores; más concretamente, tratamos de analizar algunas variables que influyen en la precisión de la estimación del coeficiente de asociación, que no fue estudiado por ellos. 


\section{Método}

La muestra estuvo formada por un total de de 414 alumnos de primer año de la Licenciatura en Psicología (62 de la Universidad de Huelva, 237 de la Universidad de Granada y 115 de la Universidad de Almería), que cursaban la asignatura obligatoria Análisis de Datos I. Aunque, todavía no habían estudiado el tema de las tablas de contingencia, los alumnos han estudiado estadística y probabilidad en la Educación Secundaria y Bachillerato. El 52\% provenían del Bachillerato de Ciencias Sociales y el 30,3\% de Ciencias de la salud, en los cuáles han estudiado estadística en el último curso.

El cuestionario utilizado está adaptado de Estepa y Batanero (1995), variando el contexto para hacerlo más adecuado a los estudiantes de psicología, y aumentando las frecuencias en todas las celdas, con objeto de que fuese posible aplicar el cálculo del estadístico Chi-cuadrado. El resto de las variables toman el mismo valor que en los ítems originales, para facilitar la comparación de nuestro trabajo con el citado (Figura 1).

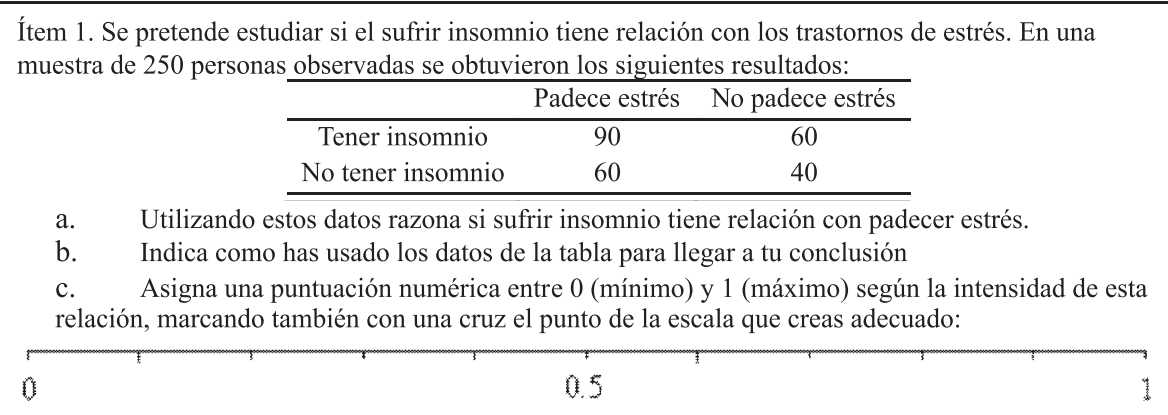

Figura 1 - Ejemplo de ítem

En la Figura 1 se presenta el primero de los ítems. El cuestionario completo se incluye como anexo. Las variables de tarea tenidas en cuenta en el cuestionario (Tabla 2) fueron las siguientes:

1. Signo de la asociación entre las variables, considerándose los tres casos posibles: Dependencia directa, dependencia inversa e independencia.

2. Intensidad de la dependencia, medida mediante el coeficiente Phi de Pearson en tablas $2 \times 2$ y con el coeficiente V de Cramer en tablas $2 \times 3$. Se eligió un ítem de intensidad moderada-baja y dos de intensidad moderada-alta.

3. Concordancia entre los datos y las teorías previas sugeridas por el contexto. Se usaron dos ítems en que coincide la asociación empírica en los 
datos con las expectativas previas, otro donde no coincide y uno donde no hay teorías previas.

4. Tipo de covariación. Usamos tres categorías consideradas por Barbancho (1973): Dependencia causal unilateral, interdependencia y dependencia indirecta.

Tabla 2 - Variables de tarea en los ítems

\begin{tabular}{|c|c|c|c|c|}
\hline & & Tabla $2 \times 2$ & & Tabla $2 \times 3$ \\
\hline & Item 1 & Item 2 & Item 3 & Item 4 \\
\hline Dependencia & Independencia & Inversa & Directa & Directa \\
\hline $\begin{array}{l}\text { Valor del coeficiente de } \\
\text { asociación }\end{array}$ & 0 & $-0,62$ & 0,67 & 0,37 \\
\hline Concuerda con teoría previa & No & $\mathrm{Si}$ & No hay teoría & $\mathrm{Si}$ \\
\hline Tipo covariación & Interdependencia & $\begin{array}{c}\text { Causal } \\
\text { unilateral }\end{array}$ & $\begin{array}{l}\text { Dependencia } \\
\text { indirecta }\end{array}$ & $\begin{array}{c}\text { Causal } \\
\text { unilateral }\end{array}$ \\
\hline
\end{tabular}

\section{Resultados y discusión}

Recogidos los datos, se analizaron las respuestas de los estudiantes en cada apartado. En lo que sigue se analizan los juicios de asociación de los estudiantes, intensidad estimada para el coeficiente de asociación e influencia de la universidad de procedencia, juicio de asociación y corrección de la estrategia utilizada sobre la precisión de la estimación.

\subsection{Juicio de asociación}

En el apartado (a) de cada ítem se pide a los estudiantes un juicio sobre la posible asociación entre las variables presentadas en la tabla, es decir, indicar si consideran o no que las variables están relacionadas. Para evaluar la competencia de los estudiantes al realizar estos juicios, se presenta, en la Tabla 3, el porcentaje de estudiantes que considera (o no) la existencia de relación entre las variables. Añadimos en la cuarta columna el coeficiente de asociación real calculado con los datos del ítem y en la quinta la existencia o no de teorías previas sobre la asociación en el ítem por parte de los estudiantes.

Tabla 3 - Frecuencia (y porcentaje) de juicios sobre la asociación entre las variables

\begin{tabular}{lccccc}
\hline Ítem & $\begin{array}{c}\text { Consideran } \\
\text { relación }\end{array}$ & $\begin{array}{c}\text { Consideran } \\
\text { independencia }\end{array}$ & No contestan & $\begin{array}{c}\text { Coeficiente de } \\
\text { asociación }\end{array}$ & $\begin{array}{c}\text { Teorías } \\
\text { previas }\end{array}$ \\
\hline 1 & $323(78,1)$ & $90(21,7)$ & $1(0,2)$ & 0 & En contra \\
2 & $398(96,1)$ & $14(3,4)$ & $2(0,5)$ & $-0,62$ & A favor \\
3 & $386(93,2)$ & $24(5,8)$ & $4(1)$ & 0,67 & No hay \\
4 & $402(97,1)$ & $4(1)$ & $8(1,9)$ & 0,37 & A favor \\
\hline
\end{tabular}


La mayoría de los alumnos indica la existencia de asociación en todos los ítems, con mayor frecuencia cuando ésta se confirma con los datos (ítems 2 , 3 y 4), pero, también en el ítem 1, donde los datos presentan independencia perfecta, independencia que no fue percibida claramente por los estudiantes, pues en una amplia mayoría indican que hay asociación.

Este resultado puede explicarse por el mecanismo de correlación ilusoria, descrito por Chapman y Chapman (1969), pues los estudiantes conocen que el estrés puede provocar pérdida de sueño; es un contexto muy conocido para ellos. En el estudio de Estepa (1994) sólo el 31,4\% de estudiantes indican que hay asociación en el caso de independencia perfecta, aunque los datos numéricos del ítem son exactamente los mismos en las dos investigaciones. La diferencia entre nuestro ítem y el de Estepa es el contexto, más relacionado con el campo de la psicología en nuestra investigación (las variables consideradas por el autor fueron fumar y tener trastornos bronquiales). Nuestros estudiantes muestran un mayor efecto de las teorías previas en este ítem, así como la concepción causal de la asociación, ligando los conceptos de asociación y causalidad. Los estudiantes manifestaron estas teorías previas y razonamientos causales en sus respuestas, como los ejemplos mostrados a continuación:

Si puede tener algo de relación, puesto que padecer estrés puede ser una causa de tener insomnio ya que las preocupaciones y de más elementos influyen", (Estudiante $52)$

En mi opinión tener insomnio si tiene relación con padecer estrés, ya que la mayoría de personas que tienen insomnio padecen estrés (Estudiante 112);

Sí tiene relación, pues las personas que padecen insomnio no descansan bien y les causa un estrés extra al que habría que sumar el que puede ocasionarte otra serie de factores externos (Estudiante 214).

Nuestros resultados son mucho mejores en el ítem 2 (asociación inversa), que en el estudio de Estepa, donde sólo el 47,1\% de estudiantes consideran la existencia de asociación. El cambio en este ítem es el contexto, pues Estepa (1994) usó las variables "seguir una dieta blanda" y "tener molestias digestivas". También en este ítem se aumentó el tamaño de muestra y la intensidad del coeficiente de asociación (-0,44 en el estudio original). Todos estos cambios han 
llevado a que apenas se haya presentado en nuestros estudiantes la concepción unidireccional de la asociación descrita por Estepa, facilitada su detección por la familiaridad del contexto, el hecho de que las teorías previas apoyan los datos y la mayor intensidad de la asociación.

Los resultados en el ítem 3 son muy similares en nuestro caso, aunque algo mejores (88,2\% en Estepa), donde sólo se aumentó las frecuencias, sin variar ni el contexto ni la intensidad de asociación. En este ítem la asociación se detecta fácilmente al ser directa e intensa y no tener los estudiantes teorías previas al respecto. Finalmente, mejoran también un poco los resultados en el ítem 4 (88,2\% de estudiantes consideran asociación en el estudio de Estepa), donde el contexto es muy conocido para el estudiante, las teorías previas apoyan a los datos y se aumentó un poco la intensidad de la asociación (manteniendo fijas el resto de variables).

En la Tabla 4 se presenta el porcentaje de estudiantes que considera asociación, según universidad, observando que los resultados son muy similares en todas ellas. Para comprobar si las diferencias son o no estadísticamente significativas se realizó un contraste de homogeneidad de muestras Chi- cuadrado, obteniendo un valor $C h i=0,99$ con 6 g.l, lo que corresponde a un valor $p=0,9861$. Por tanto, los resultados no son estadísticamente significativos y más aún el valor $p$ tan cercano a la unidad indica que las muestras son homogéneas en cuanto a los juicios de asociación, de lo que se deduce que los resultados no dependen de la Universidad de procedencia del estudiante.

Tabla 4 - Frecuencia (y porcentaje) de alumnos que consideran asociación según universidad

\begin{tabular}{lccccc}
\hline Ítem & $\begin{array}{c}\text { Universidad de } \\
\text { Huelva } \\
(\mathrm{n}=62)\end{array}$ & $\begin{array}{c}\text { Universidad de } \\
\text { Granada } \\
(\mathrm{n}=237)\end{array}$ & $\begin{array}{c}\text { Universidad de } \\
\text { Almería } \\
(\mathrm{n}=115)\end{array}$ & $\begin{array}{c}\text { Coeficiente de } \\
\text { asociación }\end{array}$ & $\begin{array}{c}\text { Teorías } \\
\text { previas }\end{array}$ \\
\hline 1 & $42(67,7)$ & $194(81,9)$ & $87(75,7)$ & 0 & En contra \\
2 & $60(96,8)$ & $230(97,1)$ & $108(93,9)$ & $-0,62$ & A favor \\
3 & $53(85,5)$ & $226(95,4)$ & $107(93,1)$ & 0,67 & No hay \\
4 & $57(91,9)$ & $235(99,2)$ & $110(95,7)$ & 0,37 & A favor \\
\hline
\end{tabular}

\subsection{Estimación del coeficiente de asociación}

En el apartado (c) de cada ítem se pedía al estudiante una puntuación entre 0 y 1 , según la intensidad que ellos percibiesen en la asociación entre las variables. La exactitud en la estimación de este coeficiente sólo fue analizada por Estepa (1994) en una muestra piloto de 50 estudiantes, pero no en su estudio 
definitivo. Tampoco analizó la influencia de la universidad de procedencia, juicio de asociación o estrategia seguida.

Hemos codificado, mediante un valor numérico, la intensidad estimada por los estudiantes. En algunos casos, ellos han indicado el valor numérico; en el resto de los casos hemos transformado el punto dibujado en la escala proporcionada a un valor numérico, midiendo con una regla milimetrada la distancia al origen y transformando la escala convenientemente. En la Tabla 5 presentamos el valor medio obtenido en el conjunto de la muestra, y en cada una de las universidades. Dicho valor sería una estimación del coeficiente de asociación (no se solicitó especificar el signo).

Tabla 5 - Estimación del coeficiente de asociación

\begin{tabular}{ccccccc}
\hline Ítem & $\begin{array}{c}\text { Valor medio } \\
\text { estimado en } \\
\text { Huelva }\end{array}$ & $\begin{array}{c}\text { Valor medio } \\
\text { estimado en } \\
\text { Granada }\end{array}$ & $\begin{array}{c}\text { Valor medio } \\
\text { estimado en } \\
\text { Almería }\end{array}$ & $\begin{array}{c}\text { Valor medio } \\
\text { estimado en } \\
\text { Total }\end{array}$ & $\begin{array}{c}\text { Coeficiente de } \\
\text { asociación }\end{array}$ & $\begin{array}{c}\text { Teorías } \\
\text { previas }\end{array}$ \\
\hline 1 & 0,44 & 0,47 & 0,51 & 0,47 & 0 & En contra \\
2 & 0,73 & 0,72 & 0,78 & 0,73 & $-0,62$ & A favor \\
3 & 0,68 & 0,68 & 0,75 & 0,70 & 0,67 & No hay \\
4 & 0,81 & 0,81 & 0,84 & 0,82 & 0,37 & A favor \\
\hline
\end{tabular}

La mayor precisión se da en la estimación del ítem 3, donde los alumnos no tienen teoría al respecto, pues el valor medio estimado es prácticamente idéntico al empírico. Hay una sobrestimación del coeficiente en los otros tres ítems para ambas provincias y la muestra global, observándose el efecto de las teorías previas de los estudiantes.

Como podemos observar, los alumnos proporcionan siempre un coeficiente de asociación alto o moderado, por el fenómeno, ya descrito de correlación ilusoria. En el ítem 1, que corresponde al caso de independencia perfecta, el valor medio estimado osciló entre 0,44 (Huelva) y 0,51 (Almería), obteniéndose un valor medio global estimado de 0,47 , aunque, de hecho, al haber independencia en los datos el verdadero valor del coeficiente es 0 .

Por otro lado, en este ítem la mayor frecuencia aparece en la celda (a), es decir la primera de las frecuencias absolutas de la tabla que corresponde a la presencia de los dos caracteres y que, según Arkes y Harkness (1983), tiene mayor impacto sobre la atención que las otra celdas. Todo ello podría haber influido en la estimación de una asociación no existente en los datos.

Para el ítem 2, correspondiente a una dependencia inversa, cuyo coeficiente de asociación tiene un valor absoluto próximo al del ítem 3 (cuya dependencia es directa), se estima un coeficiente de asociación próximo al 
empírico en todas las Universidades, algo inferior en Granada. De este modo, en nuestros alumnos no aparece un porcentaje apreciable de concepción unidireccional descrita por Estepa (1994), ni en cada muestra parcial, ni en el global, pues además, la mayoría de los alumnos indica que hay asociación (no se pidió el signo de la asociación). Este mejor resultado puede deberse a que las teorías previas estaban a favor de la asociación. Nuestros resultados contradicen el estudio de Erlick y Mills (1967) quien indica que la asociación negativa se estima como muy próxima a cero.

En el caso de dependencia directa (ítem 3), los alumnos se aproximan casi perfectamente al coeficiente de asociación. En este ítem no hubo teorías previas sobre el tema y los alumnos se guiaron más por la observación de los datos, que muestran con facilidad la dependencia, ya que seleccionamos un coeficiente de asociación alto. Estos resultados coinciden con los de Jennings, Amabile y Ross (1982), Wright y Murphy (1984), Alloy y Tabacnick (1984) y Meiser y Hewstone (2006), quienes indican que la estimación de la asociación es más precisa si las personas no tienen teorías respecto al tipo de asociación sobre los datos.

En el ítem 4, correspondiendo a una tabla 2x3 con una asociación positiva, se puede observar la gran diferencia que hay entre la estimación y su valor verdadero. Los estudiantes sobrestiman la asociación, guiándose por sus teorías previas que coinciden con el tipo de asociación en los datos. Estas teorías se manifestaron en los estudiantes al ser un contexto tan cercano a ellos (horas de estudio-aprobar o suspender), se han dejado llevar por su experiencia personal. Esto ocurre en todas las Universidades. En general, comparando los ítems donde hay teorías previas, vemos que los resultados sobrestiman el valor del coeficiente, siempre que hay una teoría sobre la existencia de asociación (ítem 1, 2 y 4) mientras dan una estimación casi perfecta cuando no tienen teorías, de acuerdo con lo que indican Chapman y Chapman (1969).

Los estudiantes de Granada y Huelva, en todos los ítems, estiman en promedio un coeficiente más bajo que los estudiantes de Almería. Al realizar un contraste $t$ de comparación de medias relacionadas, sin embargo, no obtuvimos diferencias estadísticamente significativas para ninguno de los ítems y los intervalos de confianza del $95 \%$ cubrieron el valor 0 . Asimismo, se realizaron contrastes $F$ para comparar las desviaciones típicas de cada par de variable, y tampoco se obtuvo diferencia estadísticamente significativa ni valores apreciables en el intervalo de confianza del $95 \%$ para el cociente de varianzas. De todo ello se deduce que las respuestas de los estudiantes fueron similares en su precisión de la estimación, a pesar de la diferencia de contexto educativo. 


\subsection{Consistencia entre juicio de asociación y estimación del coeficiente}

Una pregunta es si la estimación que realizan los estudiantes del coeficiente de asociación es consistente con su percepción subjetiva de la asociación, que viene dada por su juicio de asociación positivo o negativo en el ítem. Para estudiar esta consistencia, en la Tabla 6 se presentan los valores medios y error típico del coeficiente estimado en cada ítem, según el juicio de asociación haya sido positivo (se indica que hay asociación en los datos) o negativo (se niega la asociación). Asimismo, se presentan las diferencias de valores medios, junto con el error típico de la diferencia de medias, el valor $t$ en el contraste de diferencias de medias en muestras independientes y el valor $p$ correspondiente.

Tabla 6 - Estimación de la asociación según consideran o no asociación en los datos

\begin{tabular}{ccccccccc}
\hline Ítem & \multicolumn{4}{c}{ Consideran asociación } & Diferencia & $\begin{array}{c}\text { E. típico } \\
\text { diferencia }\end{array}$ & $\mathrm{t}$ & $\mathrm{p}$ \\
\cline { 2 - 5 } & \multicolumn{2}{c}{ Si } & \multicolumn{2}{c}{ No } & & & \\
\cline { 2 - 5 } & Media & E. Típico & Media & E. Típico & & & & \\
\hline 1 & 0,56 & 0,01 & 0,18 & 0,02 & 0,38 & 0,02 & 17,00 & 0,000 \\
2 & 0,75 & 0,01 & 0,31 & 0,55 & 0,44 & 0,05 & 8,76 & 0,000 \\
3 & 0,73 & 0,01 & 0,22 & 0,04 & 0,51 & 0,04 & 12,10 & 0,000 \\
4 & 0,82 & 0,01 & 0,31 & 0,08 & 0,51 & 0,07 & 6,89 & 0,000 \\
\hline
\end{tabular}

Observamos una diferencia estadísticamente muy significativa en todos los ítems, y un valor medio siempre superior en el grupo de estudiantes que da un juicio positivo de asociación, que en el grupo de los que dan un juicio negativo. Consecuentemente, hay una alta consistencia entre el juicio de asociación y el valor estimado del coeficiente, que es siempre más alto (correspondiendo a valores de correlación alta o moderada) en los estudiantes que perciben asociación en los datos. Por el contrario, los valores medios de los coeficientes corresponden a asociaciones bajas en los que dan un juicio negativo.

\subsection{Consistencia entre corrección de la estrategia y estimación del coeficiente}

Por otro lado, se ha analizado la existencia de diferencias en la estimación de la asociación, según el tipo de estrategia que emplean los estudiantes (incorrecta, parcialmente correcta o correcta). Son estrategias correctas aquellas que utilizan todos los datos de la tabla mediante la comparación de probabilidades, por ejemplo, en el ítem 4 un estudiante indica: 
Para menos de 5 horas: el $20 \%$ aprueba y el $80 \%$ suspende; Entre 5 y 10 horas: el $66 \%$ aprueba y el $33 \%$ suspende; Para más de 10 horas: el $96 \%$ aprueba y el $3 \%$ suspende. En la gráfica se observa claramente como a medida que aumenta las horas de estudio aumenta el número de aprobados (Estudiante 87).

Serían parcialmente correctas las estrategias de los estudiantes que utilizan todos los datos del problema, pero, o bien realizan comparaciones aditivas, o bien trabajan con frecuencias absolutas y no con relativas, de modo que no se cuantifican adecuadamente las probabilidades. Con estas estrategias, en algunos casos, se llega a un juicio correcto de asociación. Por ejemplo, en el problema 1, un estudiante indica:

Observando la tabla se deduce que 150 personas tienen insomnio, y de ellos 90 padecen estrés; además 100 personas no lo tienen y solo 60 de ellos padece estrés. Por lo que se puede deducir según las probabilidades que si puede tener relación (Estudiante 45).

Cualquier otra estrategia sería incorrecta. Con estas estrategias nunca se llega a una solución correcta, a no ser que sea de forma casual. Entre la variedad de estrategias incorrectas encontradas, las más frecuentes son utilizar una sola celda, estrategia considerada por Inhelder y Piaget (1955) como propia del periodo de operaciones concretas y no al de las operaciones formales. Generalmente, se utiliza la celda que corresponde a presencia de dos caracteres en la tabla $2 \times 2$ o la celda de mayor frecuencia en la tabla $2 \times 3$, como el siguiente caso (ítem 4):

100 personas: 51 aprobaron con más de 10 horas de estudio (Estudiante 236).

Ello es consistente con los resultados de Inhelder y Piaget y de autores posteriores que analizaron estas estrategias (por ejemplo, JENKINS; WARD, 1965).

En la Tabla 7 presentamos la frecuencia de estas estrategias, que fueron analizadas con detalle en un estudio previo (CAÑADAS et al., 2011), en cada uno de los ítems. Fueron muy pocas las estrategias correctas; apenas un 10\% del total o incluso menos en algunos ítems. Las estrategias parcialmente correctas a veces proporcionan un juicio de asociación correcto, pero no siempre; si se 
suman a las anteriores llegan a superar el 30 o $40 \%$ en todos los ítems en incluso se acercan al $60 \%$ en el último, que tiene un contexto muy conocido por los estudiantes. No obstante, todavía hay un gran número de estrategias incorrectas, especialmente en el primer ítem, lo que también explica la mala estimación del coeficiente en el mismo.

Tabla 7 - Porcentaje de estrategias, según su corrección en los juicios de asociación

\begin{tabular}{ccccc}
\hline Ítem & E. incorrectas & E. p. correctas & E. correctas & No responde \\
\hline 1 & $276(65,5)$ & $83(20)$ & $46(11,1)$ & $14(3,4)$ \\
2 & $249(60,2)$ & $120(29)$ & $37(8,9)$ & $8(1,9)$ \\
3 & $256(61,8)$ & $104(25,1)$ & $28(6,8)$ & $26(6,3)$ \\
4 & $157(37,9)$ & $208(50,3)$ & $27(6,5)$ & $22(5,3)$ \\
\hline
\end{tabular}

En la Tabla 8 se presentan los valores medios y error típico del valor estimado del coeficiente de asociación, en función del tipo de estrategia utilizada (correcta, parcialmente correcta o incorrecta). Una vez comprobada previamente que la homogeneidad de varianzas en las tres muestras y en cada ítem permitía llevar a cabo el análisis de varianza, así como el resto de supuestos del método (independencia, normalidad aproximada de la variable) se procedió a realizar el análisis de varianza de una vía, presentándose en la tabla el valor $p$ correspondiente a dicha prueba y en la última columna el valor empírico del coeficiente de asociación.

Tabla 8 - Estimación de la asociación según el tipo de estrategia utilizada

\begin{tabular}{ccccccccc}
\hline Ítem & $\begin{array}{c}\text { Sig. } \\
\text { Anova }\end{array}$ & \multicolumn{2}{c}{ E. incorrectas } & \multicolumn{2}{c}{ E. p. correctas } & \multicolumn{2}{c}{ E. correctas } & Coeficiente \\
\cline { 3 - 7 } & & Media & $\begin{array}{c}\text { Error } \\
\text { típico }\end{array}$ & Media & $\begin{array}{c}\text { Error } \\
\text { típico }\end{array}$ & Media & $\begin{array}{c}\text { Error } \\
\text { típico }\end{array}$ & \\
\hline 1 & 0,000 & 0,536 & 0,012 & 0,432 & 0,024 & 0,174 & 0,038 & 0 \\
2 & 0,048 & 0,728 & 0,013 & 0,717 & 0,020 & 0,810 & 0,025 & $-0,62$ \\
3 & 0,209 & 0,693 & 0,015 & 0,727 & 0,021 & 0,756 & 0,029 & 0,67 \\
4 & 0,001 & 0,785 & 0,015 & 0,846 & 0,009 & 0,797 & 0,008 & 0,37 \\
\hline
\end{tabular}

Dicho valor $p$ resultó estadísticamente significativo en todos los ítems, excepto para el ítem 3, correspondiente a la asociación directa e intensa y en el cuál los alumnos no tenían teorías previas al respecto. Como hemos visto la precisión de la estimación fue muy buena en este caso, por lo que los alumnos, incluso con una estrategia no correcta llegaron a una estimación bastante precisa del coeficiente. Para el resto de los ítems, dado que el resultado fue estadísticamente significativo, se estudiaron las diferencias entre los tres valores medios del coeficiente, correspondientes a las estrategias correctas, parcialmente 
correctas e incorrectas. Para saber entre que grupos existen las diferencias se realizaron las pruebas post hoc de Tukey obteniéndose los siguientes resultados:

- Todos los pares de diferencias fueron estadísticamente significativas (al nivel 0,001) en el ítem 1, donde observamos como el valor del coeficiente se acerca más a 0 (verdadero valor) conforme mejora la estrategia (cuando pasa de incorrecta, a parcialmente correcta y correcta). En consecuencia, hay una fuerte influencia en este ítem de la corrección de la estrategia para la detección de la independencia; mientras que los estudiantes que utilizaron estrategias incorrectas o parcialmente correctas se guían más por sus teorías previas y sobrestiman el coeficiente de asociación, los que usan estrategias correctas se guían más por los datos y estiman un coeficiente más ajustado al valor verdadero.

- No hay un patrón claro de influencia de las estrategias sobre la precisión de la estimación en los otros ítems: Por un lado, en el ítem 2 únicamente se diferencian las estrategias correctas del resto y ello tan sólo a nivel $\mathrm{p}=0,43$. Pero, en este caso, las estrategias correctas llevan a una mayor sobrestimación del coeficiente, aunque no demasiado grande. Por otro, en el ítem 4 solamente hay diferencias entre las estrategias incorrectas y parcialmente correctas (a nivel $\mathrm{p}=0,01)$ y también en este caso mejor estrategia lleva a mayor sobrestimación.

En definitiva en la identificación de la independencia (ítem 1) la corrección de la estrategia mejora dicha identificación, no mostrándose una clara influencia en el resto de los ítems.

\section{Conclusiones e implicaciones para la enseñanza de la estadística}

Los resultados muestran que los estudiantes en su mayoría juzgan la asociación, incluso en los casos en que ésta no existe, observándose el fenómeno de la correlación ilusoria y el efecto de las teorías previas sobre la precisión en la estimación del coeficiente de asociación, especialmente al juzgar la independencia, siendo el valor más preciso cuando no existen teorías previas respecto a la asociación entre las dos variables presentadas. Aunque en este trabajo nos hemos centrado en estudiantes de psicología, puesto que la evaluación se realiza al comienzo del curso, donde no se ha hecho un estudio formal del tema, podemos esperar un razonamiento muy semejante en otros estudiantes 
que ingresan en la universidad en diversas especialidades de ciencias sociales, ya que su formación secundaria será similar a los participantes en nuestro estudio.

Respecto a las concepciones descritas por Estepa (1994) se ha observado la concepción causal, pero no la unidireccional, favorecidos los resultados por el contexto más conocido y la mayor intensidad del coeficiente. Una implicación para la enseñanza es la importancia de utilizar contextos próximos al estudiante, que aumenten su motivación y faciliten su comprensión de las tareas. La estimación del coeficiente de asociación mejora si comparamos con los resultados de Estepa (1994), en todos los ítems, salvo en este caso de independencia. Es precisamente en este caso donde mayor influencia tiene el uso de estrategias formalmente correctas para mejorar la estimación del coeficiente. Será importante, entonces, que el profesor ayude a desarrollar este tipo de estrategias en los estudiantes, para facilitarle los juicios de asociación en su vida profesional y cotidiana. Los resultados son similares en las tres universidades participantes, observándose, asimismo, la alta consistencia entre juicio emitido y valor estimado del coeficiente. En consecuencia, es de esperar resultados semejantes si se repite la investigación en otros contextos.

Según Schield (2006), una persona culta debiera poder leer críticamente las tablas que encuentra en la prensa, Internet, medios de comunicación, y trabajo profesional. Esto supone no sólo la lectura literal, sino identificar las tendencias y variabilidad de los datos, lo que incluye la emisión correcta de un juicio de asociación y la estimación de su intensidad. Nuestro estudio contribuye a señalar necesidades educativas al respecto, y variables que afectan las tareas, que deberían ser tenidas en cuenta en la enseñanza.

Como hemos indicado en la introducción, estas tablas son un instrumento importante para facilitar el razonamiento estadístico en diferentes contextos (WILD; PFANNKUCH, 1999) y en su futuro trabajo, tanto los psicólogos como otros estudiantes universitarios se enfrentarán con frecuencia a este tipo de tareas, en la lectura de revistas científicas de su especialidad y en la toma de decisión (DÍAZ; GALLEGO, 2006). Un objetivo importante en la enseñanza de la asociación será ayudar a los estudiantes a superar sus sesgos de razonamiento, para mejorar su precisión en el razonamiento sobre datos bivariados y su comprensión de la utilidad de las técnicas estadísticas en la toma de decisiones adecuadas.

Todas estas razones indican la necesidad de continuar la investigación en didáctica de la matemática al respecto, para comprender mejor las variables que guían los juicios de asociación de los estudiantes y las acciones didácticas 
que debamos tomar para mejorar dichos juicios. Puesto que la concepción causal y la correlación ilusoria no parecen mejorar con una enseñanza tradicional (BATANERO; ESTEPA; GODINO, 1997) una línea prioritaria de investigación es el diseño de propuestas alternativas de enseñanza que incluyan actividades en que se enfrente a los estudiantes con estos sesgos y se les ayude a superarlos.

Agradecimiento: Proyecto EDU2010-14947 y beca FPU-AP2009-2807 (MCINN-FEDER) (MCINN- FEDER) y grupo FQM126 (Junta de Andalucía).

\section{Referencias}

ALLAN, L. G.; JENKINS, H. M. The effect of representations of binary variables on judgment of influence. Learning and Motivation, Amsterdam, v. 14, n.4, p. 381-405, nov. 1983.

ALLOY, L.B.; TABACHNICK, N. Assessment of covariation by humans and animals: The Joint influence of prior expectations and current situational information.

Psychological Review, Washington, v. 91, n. 1, p. 112-149, ene. 1984.

ARKES, H.R.; HARKNESS, A.R. Estimates of contingency between two dichotomous variables. Journal of Experimental Psychology: General, Washington, v. 112, n. 1, p. 117-135, mar. 1983.

BARBANCHO, A. G. Estadística elemental moderna. 4. ed. Barcelona: Ariel, 1973.

BATANERO, C.; ESTEPA, A.; GODINO, J. D. Evolution of students' understanding of statistical association in a computer based teaching environment. En: GARFIELD, J. B.; BURRILL, G. (Ed.). Research on the Role of Technology in Teaching and Learning Statistics. Minnesota, MN: International Statistical Institute, 1997. p. 191-206.

CAÑADAS, G. R.; BATANERO, C.; CONTRERAS, J. M.; ARTEAGA, P. Estrategias en el estudio de la asociación en tablas de contingencia por estudiantes de psicología.

Educación Matemática, México D.F., v. 23, n. 2, p. 5-32, ago. 2011.

CAZORLA, I. A relaçao entre a habilidades viso-pictóricas e o dominio de conceitos estatísticos na leitura de gráficos, 2002. 320f. Tese (Doutorado em Educação) Faculdade de Educação, Universidade de Campinas, Campinas, 2002.

CHAPMAN, L. J. Illusory correlation in observational report. Journal of Verbal Learning and Verbal Behavior, Amsterdam, v.6, n.1, p.151-155, Feb. 1967 
CHAPMAN, L. J.; CHAPMAN, J. P. Illusory correlation as an obstacle to the use of valid psychodiagnostic signs. Journal of Abnormal Psychology, Washington, v. 74, n. 1, p. 271-280, June 1969.

CROCKER, J. Judgment of covariation by social perceivers. Psychological Bulletin, Washington, v. 90, n. 2, p. 272-292, Sep. 1981.

DÍAZ, J.; GALLEGO, B. Algunas medidas de utilidad en el diagnóstico. Revista Cubana de Medicina General Integrada, La Habana, v.22, n.1, p. 1-11, mar. 2006. Disponível em: <http://bvs.sld.cu/revistas/mgi/vol22_1_06/mgi07106.htm>. Acesso em: 01 nov. 2013.

ERLICK, D. E.; MILLS, R. G. Perceptual quantification of conditional dependency. Journal of Experimental Psychology, Washington, v. 73, n. 1, p. 9-14, mar. 1967.

ESTEPA, A. Concepciones iniciales sobre la asociación estadística y su evolución como consecuencia de una enseñanza basada en el uso de ordenadores. 1993. 668f. Tesis (Doctorado en Didáctica de las Matemáticas) - Departamento de Didáctica de la Matemática, Universidad de Granada, Granada, 1994.

ESTEPA, A.; BATANERO, C. Concepciones iniciales sobre la asociación estadística. Enseñanza de las Ciencias, Barcelona, v.13, n.2, p.155-170, 1995.

INHELDER, B.; PIAGET, J. De la logique de l'enfant à la logique de l’adolescent. Paris: Presses Universitaires de France, 1955.

JENKINS, H. M.; WARD, W. C. Judgment of the contingency between responses and outcomes, Psychological Monographs, Washington, v.79, n.594, p.1-17, 1965

JENNINGS, D. L. ; AMABILE, T. M.; ROSS, L. Informal covariation assessment: Databased versus theory-based judgments. En: KAHNEMAN, D.; SLOVIC, P.; TVERSKY, A. (Ed.). Judgment under uncertainty: Heuristics and biases. Nueva York: Cambridge University Press, 1982. p. 211-230.

MCKENZIE, C. R. M.; MIKKELSEN, L. A. A Bayesian view of covariation assessment. Cognitive Psychology, Amsterdam, v.54, n.1, p.33-61, Feb. 2007

MEISER T.; HEWSTONE, M. Illusory and spurious correlations: Distinct phenomena or joint outcomes of exemplar-based category learning? European Journal of Social Psychology, East Anglia, v. 36, n. 3, p. 315-336, June 2006. 
SCHIELD, M. Statistical literay survey analysis: reading graphs and tables of rates percentages. En: INTERNATIONAL CONFERENCE ON TEACHING STATISTICSICOTS, $7^{\text {th }}, 2006$, Salvador. Proceedings... Salvador: International Association for Statistical Education, 2006. Disponible en: www.stat.auckland.ac.nz/ iase/ publications/. Acceso: 13 nov. 2012.

WILD, C.; PFANNKUCH, M. Statistical thinking in empirical enquiry. International Statistical Review, Voorburg, v. 67, n. 3, p. 223-265, Dic. 1999

WRIGHT, J. C.; MURPHY, G. L. The utility of theories in intuitive statistics: the robustness of theory-based judgments. Journal of Experimental Psychology General, Washington, v. 113, n. 2, p. 301-322, June 1984.

ZIEFFLER, A. A longitudinal investigation of the development of college students' reasoning about bivariate data during an introductory statistics course. 2006. 162f. Tesis (Doctorate in Philosophy) - Department of Educational Psychology, University of Minnesota, Estados Unidos, 2006.

Submetido em Março de 2012. Aprovado em Outubro de 2012. 


\section{ANEXO. CUESTIONARIO}

1. Se pretende estudiar si el sufrir insomnio tiene relación con los trastornos de estrés. En una muestra de 250 personas observadas se obtuvieron los siguientes resultados:

\begin{tabular}{lcc} 
& Padece estrés & No padece estrés \\
\hline Tener insomnio & 90 & 60 \\
No tener insomnio & 60 & 40 \\
\hline
\end{tabular}

a. Utilizando estos datos razona si tener o no insomnio tiene relación con padecer estrés.

b. Indica como has usado los datos de la tabla para llegar a tu conclusión

c. Asigna una puntuación entre 0 (mínimo) y 1 (máximo) según la intensidad de esta relación, marcando una cruz en el punto de esta escala que creas adecuado:

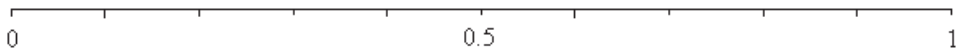

2. Un psicólogo infantil estudia si existe relación entre ser hijo único (sin hermanos) y ser un niño problemático. Para ello se han observado 250 niños obteniendo los resultados siguientes

\begin{tabular}{lcc}
\hline & Niño problemático & Niño no problemático \\
\hline Tiene hermanos & 40 & 100 \\
Hijo único & 100 & 10 \\
\hline
\end{tabular}

a. Utilizando estos datos razona si hay alguna relación entre ser un niño problemático y tener o no hermanos. conclusión.

b. Indica cómo has utilizado los números de la tabla, para llegar a tu

c. Asigna una puntuación entre 0 (mínimo) y 1 (máximo) según la intensidad de esta relación, marcando una cruz en el punto de esta escala que creas adecuado:

\begin{tabular}{l|l|l|l|l|}
\hline & 0.5 & 1
\end{tabular}

3. Se quiere saber si sufrir o no de alergia tiene relación con llevar una vida sedentaria (llevar una vida sin realizar ningún tipo de ejercicio físico). Para ello obtuvimos los siguientes resultados de 300 sujetos:

\begin{tabular}{|l|c|c|}
\hline & Sufre alergia & No sufre alergia \\
\hline Forma de vida sedentaria & 130 & 30 \\
\hline Forma de vida no sedentaria & 20 & 120 \\
\hline
\end{tabular}

a. Utilizando estos datos razona si padecer alergia tiene o no relación con llevar una vida sedentaria en estos sujetos.

b. Indica como has usado los números de la tabla, para obtener tu conclusión.

c. Asigna una puntuación entre 0 (mínimo) y 1 (máximo) según la intensidad de esta relación, marcando una cruz en el punto de esta escala que creas adecuado:

0.5

4. La siguiente tabla nos indica el número de estudiantes que aprobaron o suspendieron un examen teniendo en cuenta el tiempo que cada estudiante dedicó a prepararlo

\begin{tabular}{|l|c|c|c|c|}
\hline & Menos de $5 \mathrm{~h}$ & Entre $5 \mathrm{y} 10 \mathrm{~h}$ & Más de $10 \mathrm{~h}$ & TOTAL \\
\hline APROBADOS & 5 & 15 & 51 & 71 \\
\hline SUSPENSOS & 20 & 7 & 2 & 29 \\
\hline TOTAL & 25 & 22 & 53 & 100 \\
\hline
\end{tabular}

a. Utilizando estos datos razona si aprobar o suspender el examen tiene relación con el tiempo dedicado a prepararlo.

b. Indica como has usado los números de la tabla, para llegar a tu conclusión.

c. Asigna una puntuación entre 0 (mínimo) y 1 (máximo) según la intensidad de esta relación, marcando una cruz en el punto de esta escala que creas adecuado:

0.5

